УДК 621.313.282.2: 681.5.015.7

\title{
НЕЛИНЕЙНОЕ АЛГЕБРАИЧЕСКОЕ ОЦЕНИВАНИЕ ИНДУКТИВНОСТИ ВИБРАЦИОННОГО ЭЛЕКТРОМАГНИТНОГО АКТИВАТОРА ПО КРИВОЙ ЗАТУХАНИЯ ТОКА
}

\author{
Глазырин Александр Савельевич1,2, \\ asglazyrin@tpu.ru
}

\section{Аникин Василий Владимировичз, v-anikin2012@mail.ru}

\section{Буньков Дмитрий Сергеевич1, bunkovds@tpu.ru}

\author{
Антяскин Дмитрий Ильич1, \\ antyaskin.dmitriy@gmail.com
}

\section{Старцева Юлия Николаевна 1 , joulypaxomenko@mail.ru}

\section{Ковалев Владимир Захарович², vz_kovalev@mail.ru}

\section{Хамитов Рустам Нуриманович 3,4 , apple_27@list.ru}

\section{Кладиев Сергей Николаевич1, kladiev@tpu.ru}

\author{
Филипас Александр Александрович ${ }^{1}$, \\ filipas@tpu.ru \\ 1 Национальный исследовательский Томский политехнический университет, \\ Россия, 634050, г. Томск, пр. Ленина, 30 \\ 2 Югорский государственный университет, \\ Россия, 628012, г. Ханты-Мансийск, ул. Чехова, 16. \\ 3 Омский государственный технический университет, \\ Россия, 644050, г. Омск, пр. Мира, 11. \\ 4 Тюменский индустриальный университет, \\ Россия, 625000, г. Тюмень, ул. Володарского, 38.
}

\begin{abstract}
Актуальность. В технологиях, связанных с подготовкой буровых растворов и разжижением высоковязких нефртепродуктов, перспективно применение вибрационных электромагнитных активаторов. Вибрационные электромагнитные активаторы, работающие на околорезонансных частотах в предельных безударных режимах с настройкой на максимальную энергоэфрфективность, требуют определения индуктивности катушек при ффиксированных значениях магнитного зазора. Одним из наиболее перспективных способов определения индуктивности является предварительная идентификация параметров настраиваемой модели по кривым затухания тока.

Цель: разработать способ идентификации индуктивности катушки вибрационного электромагнитного активатора при фиксированной величие магнитного зазора на основе регрессионного анализа свободной составляющей тока.

Методы: обыкновенные диффреренциальные уравнения, прямое преобразование Лапласа при ненулевых начальных условиях, передаточные функции, импульсные переходные характеристики, регрессионный анализ, методы решения диффреренциальных уравнений, метод Ньютона, теория оптимизации, минимизация на основе суммы квадратов невязок целевой функции.

Результаты. Показан способ предварительной идентификации индуктивности катушек вибрационного электромагнитного активатора на основе регрессионного анализа кривой затухания тока. Выведено выражение для целевой функции и составлено нелинейное алгебраическое уравнение относительно ее производной по оцениваемому параметру. При существенном отклонении априорных значений оцениваемой величины от истинного как в большую, так и в меньшую сторону продемонстрирована работоспособность, быстрота сходимости и динамика изменения погрешности разработанного способа. При использовании 10-разрядного аналогово-цисрового преобразователя для вхождения в зону нечувствительности алгоритму предварительной идентификации индуктивности потребуется не более 11 циклов расчета, а при использовании 12 разрядного аналогово-цифрового преобразователя - не более 13 циклов расчета.
\end{abstract}

\section{Ключевые слова:}

Вибрационный электромагнитный активатор, буровой раствор, высоковязкий нефтепродукт, магнитопровод сложной формы, кривая затухания тока, оценивание индуктивности, регрессионный анализ, нелинейное алгебраическое уравнение, метод Ньютона для решения нелинейного уравнения. 


\section{Введение}

Вибрационные электромагнитные активаторы (ВЭМА), применяемые в технологиях разжижения высоковязких нефтепродуктов [1-6] и приготовления буровых растворов [7, 8], имеют магнитопровод сложной формы [9]. Главной причиной конструктивной сложности магнитной системы ВЭМА является наличие якоря активатора, имеющего дугообразные вырезы трапецеидального сечения и служащие для образования затопленных струй. Оптимальные с точки зрения энергоэффективности и производительности режимы работы ВЭМА обеспечивает система управления, описанная в российском патенте [10] и отдельных работах $[11,12]$. Для расчетов емкости конденсатора в контуре сброса, а также максимально допустимой частоты полумостового резонансного инвертора требуется тем или иным способом определить индуктивность катушек при фиксированном зазоре [9]. Рассмотрим несколько способов определения упомянутых индуктивностей. Первый способ для определения индуктивности катушки основан на решении известных уравнений Максвелла для электромагнитного поля $[13,14]$. Результат расчета магнитного поля ВЭМА на основе метода конечных элементов представлен на рис. 1.

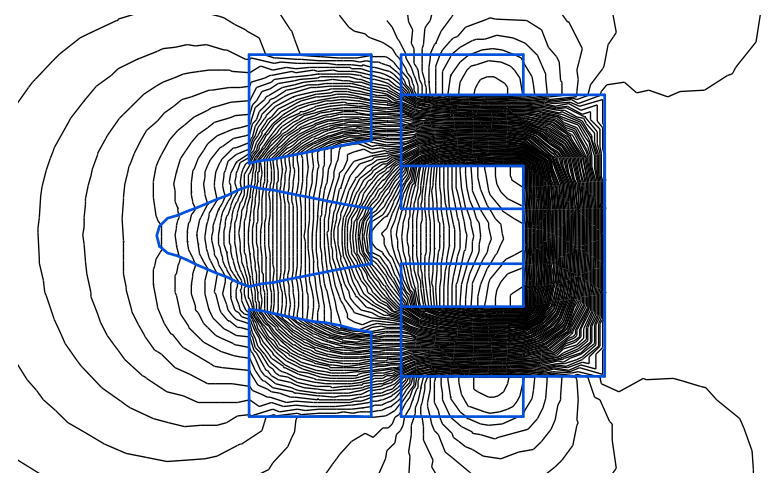

Puc. 1. Картина магнитного поля вибрационного электромагнитного активатора с магнитопроводом сложной формьг

Fig. 1. Picture of a magnetic field in vibration electromagnetic activator with a complex magnetic circuit

Как видно из рисунка, в магнитном поле зазора между якорем-активатором и неподвижным П-образным магнитопроводом есть существенно неоднородные участки. Расчет магнитного поля на основе метода конечных элементов можно проводить только на отдельном компьютере со значительным вычислительным ресурсом. Применение для этих целей микроконтроллера, непосредственно управляющего процессами вибрационного перемешивания и активации обрабатываемых сред, невозможно в силу ограниченности его вычислительных ресурсов. Величина магнитного зазора ВЭМА в положении равновесия может предварительно устанавливаться до начала работы устройства на основе априорных данных о реологических свойствах обрабатываемых жидких сред. По этой причине определять индуктивность катушек при фиксированном зазоре с помощью первого описанного способа не целесообразно. В качестве второго способа расчета индуктивностей катушек ВЭМА во всех диапазонах величин зазоров и потокосцеплений в работе [9] предлагается использовать известный метод объемных элементарных пространственных фигур магнитного поля $[15,16]$. Упрощенная пространственная модель одного канала ВЭМА с учетом основного потока, потоков выпучивания и рассеяния показана на рис. 2.
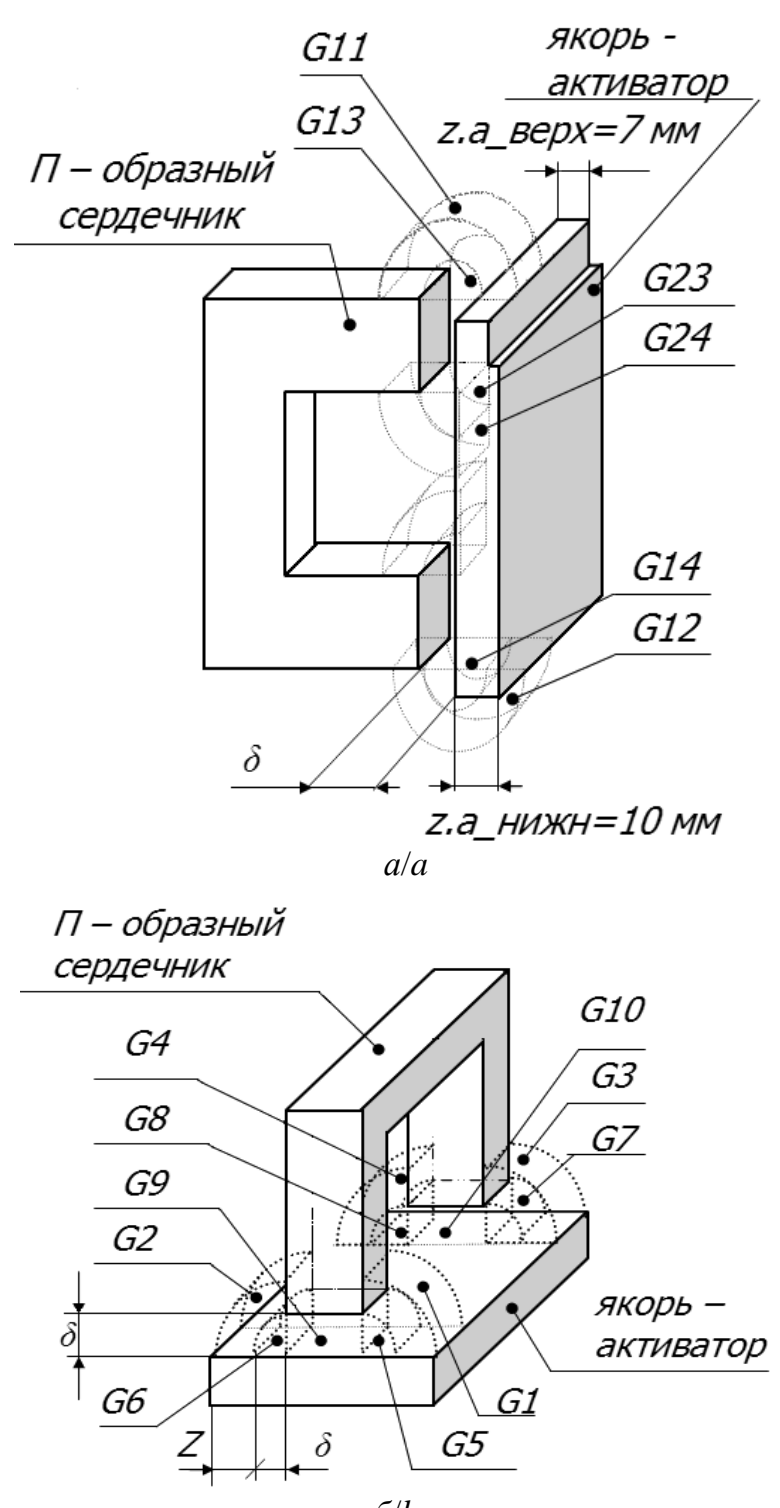

$\sigma / b$

Рис. 2. Упрощенная пространственная модель одного канала вибрационного электромагнитного активатора с учетом основного потока, потоков выпучивания и рассеяния [9], вид сбоку (а), вид cверxy (б)

Fig. 2. Simplified three-dimensional model of vibration electromagnetic activator channel taking into account the main flux, distortion and leakage flux [9], side view (a), view from above (b) 
Второй способ вычисления индуктивности некритичен к вычислительным ресурсам микроконтроллера, управляющего ВЭМА. К основному недостатку этого способа следует отнести относительно невысокую точность и существенный рост погрешности метода при максимальном зазоре между якорем-активатором ВЭМА и стенкой.

Данная статья посвящена третьему, альтернативному, способу определения индуктивности катушек ВЭМА при любом фиксированном значении зазора. Способ относится к сфере предварительной идентификации параметров динамических систем [17-22]. На первом этапе, до проведения эксперимента, необходимо жестко зафиксировать все три якоряактиватора ВЭМА в положении равновесия, величины зазоров в каждом из каналов должны быть одинаковы. Величину зазора магнитной цепи здесь и далее, на примере устройства ВЭМА- 0.3 , примем равной 0,5 мм. Это позволит обеспечить симметрию цепи нагрузки полумостового резонансного инвертора (рис. 3, a). Так как в цепь нагрузки включены 6 параллельных $R L$-цепей, три из которых представляют собой магнитосвязанные катушки, после установления одинаковых магнитных зазоров (якорь-активатор механически застопорен) в дальнейшем по умолчанию считается, что ток одной катушки получен путем домножения сигнала с датчика на масштабирующий коэффициент 1/6. В процессе накачки тока оба ключа - VT1 и VT2 - полумостового резонансного инвертора открыты (рис. 3, a).
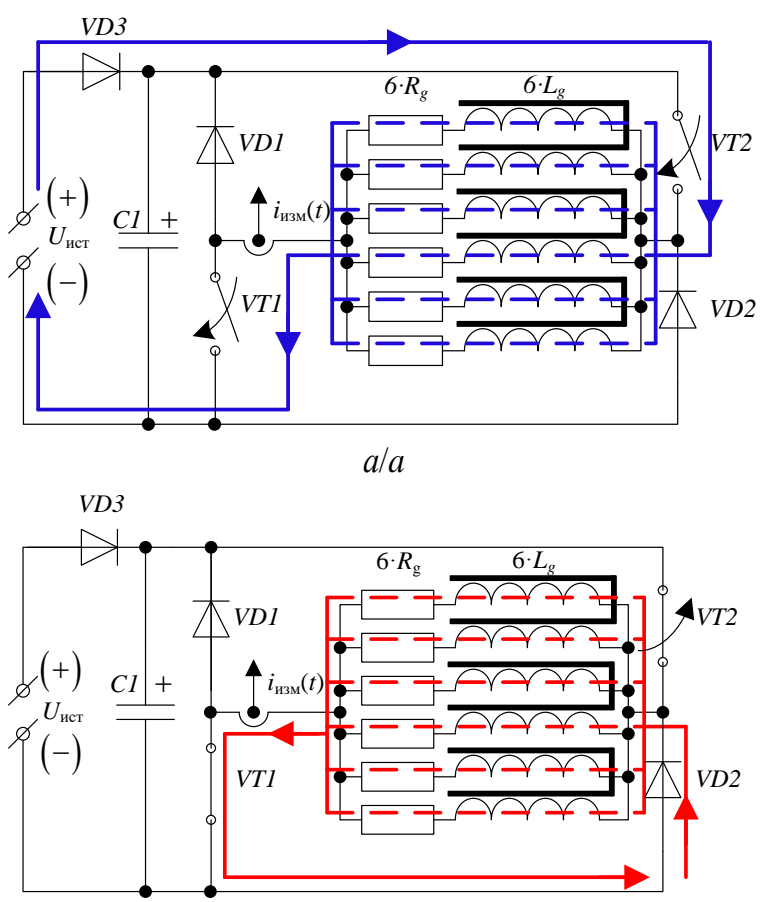

$6 / b$

Pис. 3. Схема полумостового резонансного инвертора 6 режимах накачки тока (а) и снятия апериодической кривой затухания тока (б)

Fig. 3. Half-bridge resonant inverter circuit in raising current (a) and exponential failing current curve record (b) modes
Для получения кривой затухания тока ключ VTI остается замкнутым, а ключ VT2 размыкается (рис. 3, б). Это специальный режим, не описанный в $[9,10]$. До момента коммутации в катушке фиксируется величина тока, которая была накоплена в процессе фазы накачки (рис. $3, a$ ). Начиная с момента коммутации ключа VT2, ток в катушке будет затухать по апериодическому закону, который характеризует свободную составляющую переходного процесса. Омическое сопротивление катушки измеряется отдельно и в данном эксперименте считается априорно известным. По кривой затухания тока можно определить постоянную времени $R L$-цепи или индуктивность катушки. Известны решения по определению индуктивности по кривой затухания тока, требующие вычисления производной тока на начальном участке [23]. Операция цифрового дифференцирования весьма чувствительна даже к относительно малому уровню помех в полезном сигнале, снимаемом с датчика [24]. К первому достоинству предлагаемого в данной статье способа оценивания индуктивности относится отсутствие необходимости вычисления производной тока по дискретным во времени замерам. Вторым достоинством способа является то, что математический аппарат регрессионного анализа, лежащий в основе предложенного способа, является надежным инструментом для выделения тренда полезного сигнала из аддитивной смеси сигнал-шум [24]. К третьему достоинству следует отнести то, что индуктивность определяется на основе анализа кривой затухания тока и не требует детального учета таких компонентов магнитного потока, как потоки выпучивания и рассеяния, о трудности расчета которых было отмечено во многих профильных работах $[9,15,16]$.

\section{Составление регрессионной математической модели для описания кривой затухания тока с учетом аддитивной составляющей помех в канале измерения}

Функциональная схема для построения процедуры оценивания индуктивности на основе экспоненциальной кривой затухания тока представлена на рис. 4 .

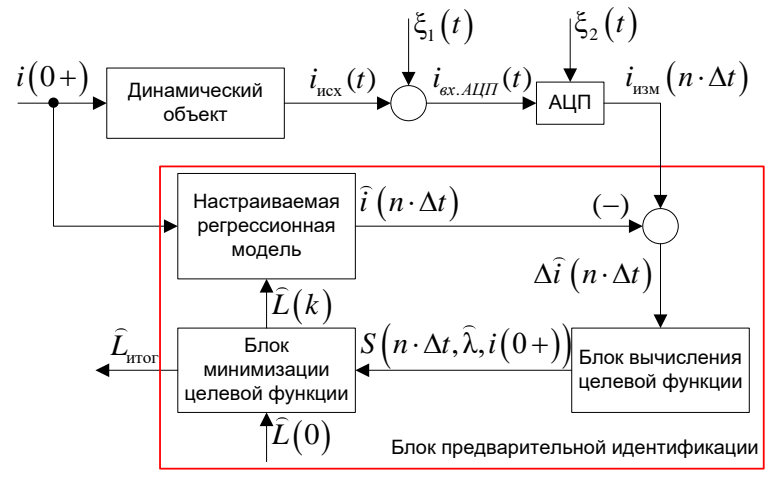

Pис. 4. Функииональная схема иифрового устройства для идентификачии индуктивности вибрационного электромагнитного активатора по кривой затухания тока

Fig. 4. Functional diagram of digital device for identification of vibration electromagnetic activator inductance by failing current curve 
Согласно функциональной схеме (рис. 4) одновременно на вход динамического объекта и настраиваемой регрессионной модели поступает сигнал $i(0+)$ - величина тока в момент коммутации ключа VT2 (рис. 3, б). Поскольку дальнейшие выкладки подразумевают использование переходных процессов затухания тока, то для аналитического описания отклика настраиваемой модели удобно применять прямое преобразование Лапласа при ненулевых начальных условиях

$$
\left.\frac{\mathrm{d} i(t)}{\mathrm{d} t}\right|_{i(0+) \neq 0} \Rightarrow p \cdot I(p)-i(0+),
$$

где $p$ - оператор Лапласа; $I(p)$ - изображение тока по Лапласу.

Реакцией динамического объекта на входное воздействие является кривая затухания тока $i_{\text {исх }}(t)$. Исходный сигнал кривой тока $i_{\text {исх }}(t)$ может быть доступен для наблюдения только в модельных задачах, в реальных системах этот сигнал необратимо смешан с помехами измерительной системы $\xi_{1}(t)$. К помехам $\xi_{1}(t)$ следует отнести помехи, вызванные несовершенством канала измерения, несовершенством регулировочной характеристики датчика (зона нечувствительности, насыщение, гистерезис). При прохождении через аналогово-цифровой преобразователь (АЦП) сигнал $i_{\text {вх.Ацп}}(t)$ подвергается двум видам преобразования: дискретизации по времени с периодом дискретизации $\Delta t$ и квантованию по уровню с добавлением аддитивной помехи квантования $\xi_{2}(t)$, зависящим от разрядности АЦП [25]. Для дальнейших выкладок сделано допущение, что суммарные помехи $\xi(t)=\xi_{1}(t)+\xi_{2}(t)$ в измерительной системе имеют форму белого шума с гауссовским распределением. На выходе измерительной системы был получен сигнал о затухании свободной составляющей тока $i_{\text {изм }}(n \cdot \Delta t)$, где $n-$ текущий шаг по времени. Невязка $\Delta \widehat{i}(n \cdot \Delta t)$ между измеренным значением тока $i_{\text {изм }}(n \cdot \Delta t)$ и откликом настраиваемой регрессионной модели $\hat{i}(n \cdot \Delta t)$ используется при расчете целевой функции $S(n \cdot \Delta t, \hat{\lambda}, i(0+))$, где $\hat{\lambda}-$ показатель быстроты затухания переходного процесса. Схема замещения после коммутации показана на рис. 3 , б. Блок минимизации целевой функции реализует итерационный расчет оценки искомой индуктивности $\widehat{L}(k)$ до достижения заданного минимума целевой функции, где $k$ - индекс итерационного процесса. Априорное значение индуктивности $\widehat{L}(0)$ задается до начала процесса вычисления. При этом точность полученной итоговой оценки $\hat{L}_{\text {итог }}$ как результат решения поставленной задачи согласуется с погрешностью квантования АЦП, применяемого в измерительной системе.

Формула, описывающая отклик $\hat{i}(t)$ настраиваемой регрессионной модели как процесс затухания тока в закороченной $R L$-цепи, имеет вид:

$$
\hat{i}(t)=\frac{U_{\text {ист }}}{R} \cdot e^{\frac{-R \cdot t}{\widehat{L}}}=i(0+) \cdot e^{\frac{-R \cdot t}{\widetilde{L}}},
$$

где $R$ - априорное значение активного сопротивления, задаваемое до начала процесса вычисления; $\hat{L}$ оценка индуктивности; $U_{\text {ист }}$ - напряжение источника постоянного тока.

Согласно (1) реакция отклика настраиваемой модели равна

$$
\hat{i}(t, \widehat{L}, i(0+))=i(0+) \cdot e^{\frac{-R \cdot t}{\widetilde{L}}} .
$$

Оцениваемый показатель быстроты затухания переходного процесса определяется

$$
\tilde{\lambda}=\frac{R}{\widehat{L}} \text {. }
$$

С учетом выражения (3) уравнение (2) принимает вид

$$
\hat{i}(t, \hat{\lambda}, i(0+))=i(0+) \cdot e^{-\hat{\lambda} \cdot t} .
$$

Невязка $\Delta \hat{i}$ с учетом (4):

$$
\begin{gathered}
\Delta \hat{i}(t, \hat{\lambda}, i(0+))=i_{\text {изм }}(t)- \\
-\hat{i}(t, \hat{\lambda}, i(0+))=i_{\text {изм }}(t)-i(0+) \cdot e^{-\hat{\lambda} \cdot t} .
\end{gathered}
$$

Предложенный алгоритм идентификации потребует вычисления первой производной от целевой функции, и для избавления от знака невязки воздержимся от применения операции вычисления модуля, так как это приведёт к проблеме неопределенности при вычислении производной от целевой функции при стремлении невязки к нулю.

Для избавления от знака невязки возведём выражение (5) в квадрат

$$
\Delta \widehat{i}(t, \hat{\lambda}, i(0+))^{2}=\left(i_{\text {изм }}(t)-\hat{i}(t, \hat{\lambda}, i(0+))\right)^{2} .
$$

После упрощения (6) получим выражение вида

$$
\begin{gathered}
\Delta \hat{i}(t, \hat{\lambda}, i(0+))^{2}=i_{\text {изм }}(t)^{2}- \\
-2 \cdot i_{\text {изм }}(t) \cdot i(0+) \cdot e^{-\hat{\lambda} \cdot t}+i(0+)^{2} \cdot e^{-2 \cdot \hat{\lambda} \cdot t} .
\end{gathered}
$$

Перейдем от непрерывного времени к дискретному

$$
t=n \cdot \Delta t .
$$

В численных примерах, иллюстрирующих в данной статье предложенный способ идентификации индуктивности, примем $\Delta t=100$ мкс, что соответствует частоте дискретизации 10 кГц. Чрезмерное снижение частоты дискретизации недопустимо, так как это вступает в противоречие с требованиями теоремы В.А. Котельникова [26, 27]. Чрезмерное повышение частоты дискретизации усложняет процесс фильтрации шумовой составляющей измеренного сигнала. Выбор частоты дискретизации 10 кГц для решения данной задачи обусловлен компромиссом между этими двумя крайностями. Плата сбора данных NI $6024 E$ обеспечивает выбранную частоту дискретизации.

Согласно выражениям (7) и (8) формула расчета невязки в дискретной форме 


$$
\begin{gathered}
\Delta \hat{i}(n \cdot \Delta t, \hat{\lambda}, i(0+))^{2}= \\
=i_{\text {изм }}(n \cdot \Delta t)^{2}-2 \cdot i_{\text {изм }}(n \cdot \Delta t) \cdot i(0+) \cdot e^{-\hat{\lambda} \cdot n \cdot \Delta t}+ \\
+i(0+)^{2} \cdot e^{-2 \cdot \hat{\lambda} \cdot n \cdot \Delta t} .
\end{gathered}
$$

Элемент суммы квадратов невязок записывается

$$
\Delta S_{n}(n \cdot \Delta t, \hat{\lambda}, i(0+))=\Delta \hat{i}(n \cdot \Delta t, \hat{\lambda}, i(0+))^{2} .
$$

Сумма квадратов невязок - целевая функция для настраиваемой модели (рис. 4):

$$
\begin{gathered}
S(n \cdot \Delta t, \hat{\lambda}, i(0+))=\sum_{n=1}^{m} \Delta S_{n}(n \cdot \Delta t, \hat{\lambda}, i(0+))= \\
=\sum_{n=1}^{m} \Delta \widehat{i}(n \cdot \Delta t, \hat{\lambda}, i(0+)) ; \\
S(n \cdot \Delta t, \hat{\lambda}, i(0+))=\sum_{n=1}^{m} i_{\text {изм }}(n \cdot \Delta t)^{2}- \\
-2 \cdot i_{\text {изм }}(n \cdot \Delta t) \cdot i(0+) \cdot e^{-\hat{\lambda} \cdot n \cdot \Delta t}+i(0+)^{2} \cdot e^{-2 \cdot \hat{\lambda} \cdot n \cdot \Delta t} .
\end{gathered}
$$

Вычислим производную $D S$ целевой функции по настраиваемому аргументу. Для удобства вычисления воспользуемся правилом, по которому производная от суммы функций равна сумме производных этих функций.

Найдём производную от квадрата невязки на шаге $n$

$$
\begin{aligned}
& \frac{\mathrm{d}}{\mathrm{d} \widehat{\lambda}}\left(i_{\text {изм }}(n \cdot \Delta t)^{2}-2 \cdot i_{\text {изм }}(n \cdot \Delta t) \cdot i(0+) \cdot e^{-\hat{\lambda} \cdot n \cdot \Delta t}+\right. \\
& \left.+i(0+)^{2} \cdot e^{-2 \cdot \hat{x} \cdot n \cdot \Delta t}\right) ; \\
& \frac{\mathrm{d}}{\mathrm{d} \hat{\lambda}}\left(i_{\text {изм }}(n \cdot \Delta t)^{2}\right) \rightarrow 0 ; \\
& \frac{\mathrm{d}}{\mathrm{d} \widehat{\lambda}}\left(-2 \cdot i_{\text {изм }}(n \cdot \Delta t) \cdot i(0+) \cdot e^{-\hat{\lambda} \cdot n \cdot \Delta t}\right) \rightarrow \\
& \rightarrow 2 \cdot n \cdot \Delta t \cdot i_{\text {изм }}(n \cdot \Delta t) \cdot i(0+) \cdot e^{-\hat{\lambda} \cdot n \cdot \Delta t} ; \\
& \frac{\mathrm{d}}{\mathrm{d} \hat{\lambda}}\left(i(0+)^{2} \cdot e^{-2 \cdot \hat{\lambda} \cdot n \cdot \Delta t}\right) \rightarrow-2 \cdot n \cdot \Delta t \cdot i(0+)^{2} \cdot e^{-2 \cdot \lambda \cdot \Delta \cdot \Delta t} ; \\
& D S\left(n \cdot \Delta t, \hat{\lambda}, i_{0}\right)=\frac{\mathrm{d}}{\mathrm{d} \widehat{\lambda}} S\left(n \cdot \Delta t, \widehat{\lambda}, i_{0}\right)= \\
& =\sum_{n=1}^{m}\left(0+2 \cdot n \cdot \Delta t \cdot i_{\text {изм }}(n \cdot \Delta t) \cdot i(0+) \cdot e^{-\hat{\lambda} \cdot n \cdot \Delta t}-\right. \\
& \left.-2 \cdot n \cdot \Delta t \cdot i(0+)^{2} \cdot e^{-2 \cdot \lambda \cdot n \cdot \Delta t}\right) .
\end{aligned}
$$

Упростим (10)

$$
\begin{gathered}
D S(n \cdot \Delta t, \hat{\lambda}, i(0+))=2 \cdot i(0+) \cdot \Delta t \times \\
\times \sum_{n=1}^{m}\left(n \cdot i_{\text {изм }}(n \cdot \Delta t) \cdot e^{-\hat{\lambda} \cdot n \cdot \Delta t}-i(0+) \cdot n \cdot e^{-2 \cdot \hat{\lambda} \cdot n \cdot \Delta t}\right) \cdot(
\end{gathered}
$$

В результате получено выражение (11) для производной целевой функции по искомому параметру в аналитическом виде, позволяющее применить известный метод Ньютона (касательных), обладающий наивысшей скоростью сходимости.
Показатель $\hat{\lambda}(k)$, согласно методу Ньютона, определяется

$$
\hat{\lambda}(k)=\hat{\lambda}(k-1)-\frac{S(n \cdot \Delta t, \hat{\lambda}(k-1), i(0+))}{D S(n \cdot \Delta t, \bar{\lambda}(k-1), i(0+))} .
$$

Согласно выражениям (9), (11) и (12) итерационный алгоритм идентификации показателя быстроты затухания $\bar{\lambda}(k)$ имеет вид:

$$
-\frac{\hat{\lambda}(k)=\hat{\lambda}(k-1)-}{\left\{\sum_{n=1}^{m}\left[\begin{array}{l}
i_{\text {изм }}(n \cdot \Delta t)^{2}-2 \cdot e^{-\hat{\lambda} \cdot n \cdot \Delta t} \cdot i(0+) \times \\
\times i_{\text {изм }}(n \cdot \Delta t)+i(0+)^{2} \cdot e^{-2 \cdot \hat{\lambda} \cdot n \cdot \Delta t}
\end{array}\right]\right\}} .
$$

При вариации $j=\overline{1,2}$ задания начального приближения согласно (3)

$$
\widehat{\lambda}_{j}(0)=\frac{R}{\widehat{L}_{j}(0)} .
$$

Отклики настраиваемой регрессионной модели согласно (4) равны $\hat{i}_{j}\left(n \cdot \Delta t, \hat{\lambda}_{j}(k), i(0+)\right)$, где $j-$ номер эксперимента аппроксимации на основе регрессионной кривой (1) при заданном начальном приближении. Отклики $\hat{i}_{j}\left(n \cdot \Delta t, \bar{\lambda}_{j}(k), i(0+)\right)$ представляют собой регрессионное восстановление кривой затухания тока $i_{\text {изм }}(t)$, полученной от измерительной системы с высоким содержанием помех (рис. 5).

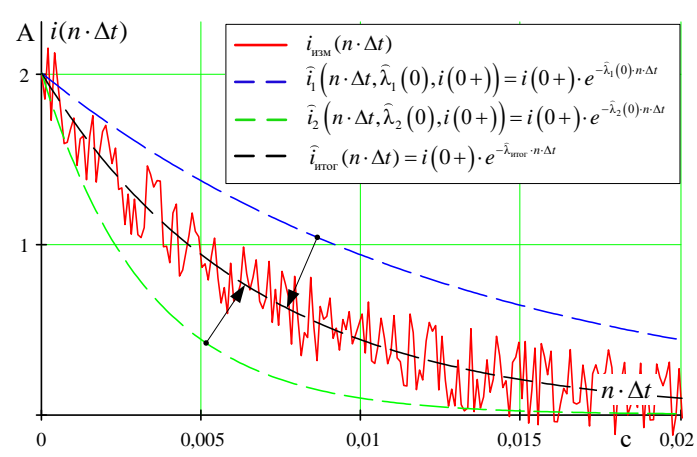

Рис. 5. Кривая измеренного сигнала, а также кривые откликов настраиваемой модели при различных значениях оценки искомого параметра

Fig. 5. Measured signal curve and response curves of customizable model at different values of searched parameter

Согласно выражению (13), было исследовано два варианта аппроксимации кривой $i_{\text {изм }}(n \cdot \Delta t)$. Синей линией показана кривая затухания тока $\hat{i}_{1}\left(n \cdot \Delta t, \hat{\lambda}_{1}(0), i(0+)\right)$ при заданном начальном приближении оценки показателя быстроты затухания переходного процесса $\hat{\lambda}_{1}(0)$, существенно превышающего искомую величину, а зеленой линией показана кривая $\hat{i}_{2}\left(n \cdot \Delta t, \hat{\lambda}_{2}(0), i(0+)\right)$ при показателе $\hat{\lambda}_{2}(0)$, существенно меньшем относи- 
тельно искомой величины. Кривая затухания тока $i_{\text {итог }}(n \cdot \Delta t)$ построена при условии, что искомая индуктивность определена абсолютно точно (без погрешностей), что оказалось возможным, так как решалась модельная задача. При идентификации индуктивности на основе реальных кривых затухания тока, полученных с платы сбора данных, например: NI 6024E, эту кривую абсолютно точно восстановить нельзя.

Искомая оценка индуктивности $\widehat{L}_{j}(k)$ на каждом шаге расчета алгоритма идентификации определена

$$
\widehat{L}_{j}(k)=\frac{R}{\widehat{\lambda}_{j}(k)} .
$$

Относительная погрешность оценивания индуктивности

$$
\Delta \widehat{L}_{j}(k)=\frac{\widehat{L}_{j}(k)-L_{\text {эталон }}}{L_{\text {эталон }}} \cdot 100 \%,
$$

где $L_{\text {эталон }}$ - эталонное значение индуктивности, которое в модельной задаче известно, $L_{\text {эталон }}=105 \mathrm{м}$ Н.

Относительная погрешность процесса квантования по уровню рассчитывается как

$$
\delta_{\text {АЦП }}=\frac{1}{2^{N_{\text {АЦП }}}} \cdot 100 \%,
$$
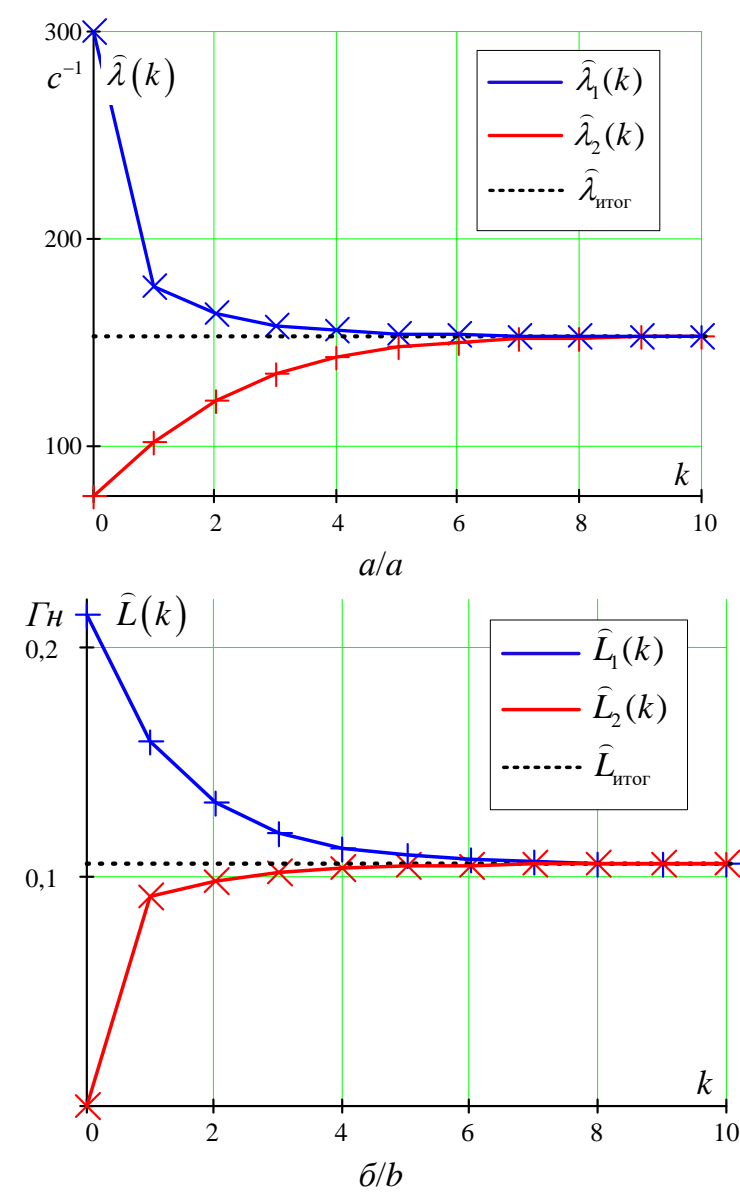

Рис. 6. Итерационные процессы оценок показателя быстроты затухания $\hat{\lambda}(k)$ (a) и индуктивности $\hat{L}(k)$ (б)

Fig. 6. Procedures of iterative search for falling rapidity (a) and inductance estimation (b) где $N_{\text {Ацп }}$ - разрядность аналогово-цифрового преобразователя. Если $N_{\text {АцП}}=10$, то

$$
\delta_{\text {АЦП }}=\delta_{10 \text { бит }}=\frac{1}{2^{10}} \cdot 100 \%=\frac{100 \%}{1024}=0,098 \% .
$$

Если $N_{\text {АцП }}=12$, то

$\delta_{\text {АЦП }}=\delta_{12 \text { бит }}=\frac{1}{2^{12}} \cdot 100 \%=\frac{100 \%}{4096}=0,024 \%$.

Итерационные процессы, иллюстрирующие работоспособность рассматриваемого алгоритма идентификации, приведены на рис. 6, 7.

Приведенные графики (рис. 6, 7) иллюстрируют высокую скорость сходимости алгоритма при определении искомой оценки индуктивности $\widehat{L}_{j}(k)$ независимо от заданных начальных приближений параметра $\hat{\lambda}_{1}(0)=3000 \mathrm{c}^{-1}$ и $\hat{\lambda}_{2}(0)=75 \mathrm{c}^{-1}$. Итерационный процесс оценивания индуктивности при $\delta_{\text {Ацп }}=\delta_{10 \text { бит }}$ войдет в зону допустимых отклонений за 8 циклов расчета для $\Delta \widehat{L}_{1}(k)$, а для $\Delta \widehat{L}_{2}(k)$ - за 11 циклов расчета. При $\delta_{\text {Ацп }}=\delta_{12 \text { бит }}$ вхождение в зону допустимых отклонений произойдет за 10 циклов расчета для $\Delta \widehat{L}_{1}(k)$, а для $\Delta \widehat{L}_{2}(k)$ - за 13 циклов расчета.

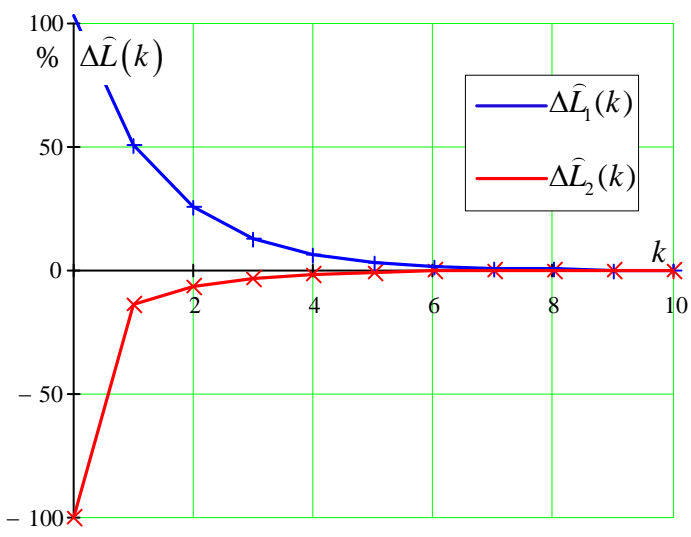

$a / a$

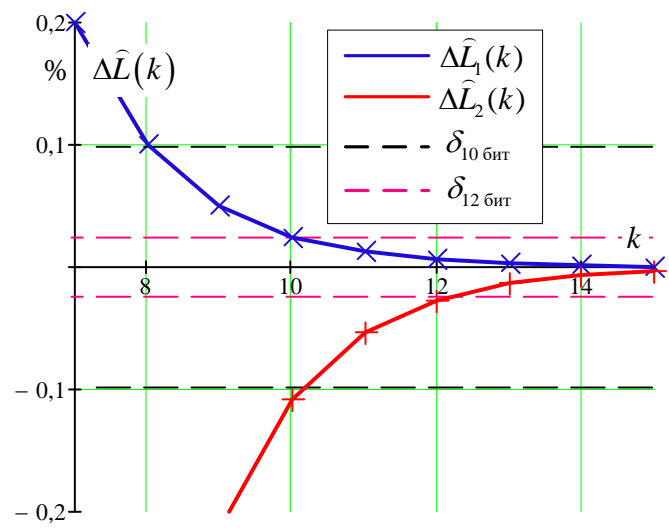

$6 / b$

Pис. 7. Итерационные процессы уменьшения относительной ошибки оченивания: вид в целом (а) и с учетом погрешности квантования 10-ти и 12-ти разрядного АЦП (б)

Fig. 7. Procedures of iterative falling of relative error for 10-bits (a) and 12-bits ADC (b) 
Для увеличения скорости сходимости алгоритма можно вести одновременный расчет сразу нескольких циклов идентификации, работающих при различных заданных начальных приближениях, усредняя на каждом шаге получаемые значения. Также увеличить скорость сходимости алгоритма позволит предварительный, более точный, выбор начальных приближений исходя из анализа кривой нарастания тока ВЭМА (рис 3,a).

\section{Выводы}

1. Показано, что для определения индуктивности катушек вибрационного электромагнитного активатора в силу повышенных вычислительных затрат затруднительно применять методы на основе решения уравнений Максвелла $[13,14]$. Метод расчета магнитных цепей ВЭМА на основе элементарных пространственных фигур магнитного поля не может дать повышенную точность расчета в силу существенной сложности конструкции магнитной системы ВЭМА. Обосновано, что в этих условиях целесообразно перейти к оцениванию индуктивности катушек ВЭМА при фиксированном зазоре на основе регрессионного анализа кривой затухания тока.

2. В работе проведено сопоставление зашумленной кривой затухания тока в катушках ВЭМА с соответствующим откликом настраиваемой регрессионной модели. Невязки между сигналом с датчика тока и, сигналом отклика настраиваемой модели возводятся в квадрат для избавления от знака не-

\section{СПИСОК ЛИТЕРАТУРЫ}

1. Pevchev V.P. Principal dimensions of the short-stroke electromagnetic motor for a seismic wave generator // Journal of Mining Science. - 2009. - V. 45. - № 4. - P. 372-381.

2. Oil recovery from tank bottom sludge using rhamnolipids / Chuhan Liu, Yin Zhang, Shanshan Sun, Lixin Huang, Li Yu, Xiaonan Liu, Ruiqiu Lai, Yijing Luo, Zhiyong Zhang, Zhongzhi Zhang // Journal of Petroleum Science and Engineering. - 2018. V. $170 .-$ P. $14-20$.

3. Analysis of oil content in drying petroleum sludge of tank bottom / Yuhua Wang, Xiaomin Zhang, Yuying Pan, Ying Chen // International Journal of Hydrogen Energy. - 2017. - V. 42. - № 29. P. $18681-18684$.

4. Da S.L., Alves F.C., De França F.P. A review of the technological solutions for the treatment of oily sludges from petroleum refineries // Waste Management \& Research. - 2012. - V. 30 (10). P. 1016-1030.

5. Lushnikov S.V., Frank Y.A., Vorobyov D.S. Oil decontamination of bottom sediments experimental work result // Earth science research journal. - 2006. - V. 10. - № 1. - P. 35-40.

6. Nekrasov V.O. Modeling bottom sediment erosion process by swirling the flow by tangential supply of oil in the tank // IOP Conference Series: Materials Science and Engineering: Transport and Storage of Hydrocarbons. - 2016. - V. 154. - 7 p. URL: https://iopscience.iop.org/article/10.1088/1757899X/154/1/012003 (дата обращения 13.06.2018).

7. Real time prediction of suspended solids in drilling fluids. Review Article / S.C. Magalhnes, C.M. Scheid, L.A. CalHada, M.M. Lutterbach, R.S. Rezende, A.T.A. Waldmann // Journal of Natural Gas Science and Engineering. - March 2016. - V. 30. P. 164-175.

8. Данекер В.А., Рикконен С.В. Приготовление и коррекция показателей бурового раствора технологией и оборудованием ВСМА // Известия Томского политехнического университета. Инжиниринг георесурсов. - 2017. - Т. 328. - № 7. - С. 86-92. вязки. Целевая функция для решения оптимизационной задачи формируется как сумма квадратов вышеупомянутых невязок, полученных для фиксированного шага дискретизации по времени на всем интервале наблюдения за кривой затухания тока. Для составления нелинейного уравнения выполнена операция дифференцирования целевой функции по искомому параметру.

3. Для решения нелинейного алгебраического уравнения применялся метод касательных (метод Ньютона). Выбор этого метода связан с быстрой его сходимостью, а также возможностью получения аналитического выражения для расчета производной от целевой функции.

4. Были продемонстрированы работоспособность, быстрота сходимости и поведение погрешности предложенного способа оценки индуктивности катушек ВЭМА при существенном расхождении начальных условий оценивания от истинного значения как в большую, так и в меньшую сторону. При 10-разрядном АЦП алгоритм затрачивает не более 11 циклов расчета, а при 12-разрядном АЦП - не более 13 циклов расчета для вхождения в зону допустимых отклонений.

5. Предложенный способ оценивания индуктивности рекомендован к применению не только в системах управления ВЭМА, но для других электромагнитных механизмов, имеющих магнитную систему сложной конфигурации.

9. Глазырин А.С. Системы питания и автоматического управления вибрационными электромагнитными активаторами: дис. ... канд. техн. наук. - Томск, 2004. - 193 с.

10. Устройство для управления электромагнитными вибраторами: пат. № 2348961 РФ. МПК G05D19/02 (2006.01). Заявка № 2008102340/28; опубл. 10.03.2008, Бюл. № 7. - 6 с.

11. Резонансные колебания с предельной амплитудой в вибрационном электромагнитном активаторе / А.Н. Гаврилин, Е.В. Боловин, А.С. Глазырин, С. Н. Кладиев, В.И. Полищук // Известия Томского политехнического университета. Инжиниринг георесурсов. - 2019. - Т. 330. - № 1. - С. 201-213.

12. Идентификация параметров механической системы вибрационного электромагнитного активатора по граничным околорезонансным частотам / А.Н. Гаврилин, С.Н. Кладиев, А.С. Глазырин, Е.В. Боловин, В.И. Полищук // Известия Томского политехнического университета. Инжиниринг георесурсов. 2019. - T. 330. - № 4. - C. 158-177.

13. Clerk Maxwell J. An elementary treatise on electricity. - Oxford: Clarendon Press, 1881. -251 p.

14. Parameters assessment of the inductively-coupled circuit for wireless power transfer / Yu.N. Isaev, O.V. Vasileva, A.A. Budko, S. Lefebvre // IOP Conference Series: Materials Science and Engineering. - 2017. - V. 177(1), no. 012097. - P. 1-6.

15. Herbert C.R. Electromagnetic Devices. - New York: John Wiley \& Sons, Inc.; London: Chapman \& Hall, Limited, 1941. - 568 p.

16. Основы теории электрических аппаратов / Б.К. Буль, Г.В. Буткевич, А.Г. Годжелло, В.Г. Кураев, Н.Е. Лысов, П.В. Сахаров, А.Г. Сливинская, И.С. Таев, А.А. Чунихин, Л.В. Шопен. - М.: Высшая школа, 1970. - 600 с.

17. Identification of parameters of power circuits pulse energy conversion systems of electromechanical equipment / E.A. Godovnikov, E.G. Andreeva, V.Z. Kovalev, R.T. Usmanov // Journal of Physics: Conference Series. - 2019. - V. 1260 (5). - № 052007. - P. 1-8.

18. Identification of mathematical models parameters of electromechanical consumers of regionally isolated electrotechnical complexes / V.Z. Kovalev, O.V. Arhipova, S.S. Esin, A.A. Tatevosyan, 
A.G. Scherbakov // Journal of Physics: Conference Series. 2019. - V. 1260 (5). -№ 052014. - P. 1-7.

19. Polishchuk V.I., Sergeev V.L. Adaptive identification method of a signal from stray magnetic field sensor for turbogenerator diagnostics // Journal of Siberian Federal University - Mathematics and Physics. - 2015. - V. 8 (2). - P. 201-207.

20. Kolomiyets E.A., Chernyshyov A.Y. Observer of rotor speed and flux is developed for the vector control system of induction motor // Proc. 2015. International Siberian Conference on Control and Communications, SIBCON 2015. - Omsk, Russia, 2015. № 7147102. - P. 1-4.

21. Experimental method for identifying high-coercive permanent magnets / A.S. Tatevosyan, A.A. Tatevosyan, V.V. Pedder, N.V. Zaharova // Journal of Physics: Conference Series. - 2019. V. 1260 (5). - № 052031. - P. 1-6.

22. Determination of equivalent circuit parameters of the electric pulse device for cleaning the surface from contamination / A.S. Tatevosyan, A.A. Tatevosyan, N.V. Zakharova, A.A. Lukacheva // Dynamics of Systems, Mechanisms and Machines: Proc. 2019, $12^{\text {th }}$ International Scientific and Technical Conference. Dynamics 2018. - Omsk, Russia, 2019. - № 8601435. - P. 1-4.

23. Ковалев А.Ю., Кузнецов Е.М., Аникин В.В. Схемотехническое исследование переходной характеристики затухания тока статора погружного асинхронного электродвигателя // Теория и практика науки третьего тысячелетия: сборник статей. Уфа: РИЦБашгу, 2014. - С. 149-152.

24. Боловин Е.В. Разработка алгебраических методов идентификации параметров асинхронных двигателей на основе дискретных моделей: дис. ... канд. техн. наук. - Томск, 2018. $271 \mathrm{c}$.

25. Козаченко В.Ф. Микропроцессоры: руководство по применению 16-разрядных микропроцессоров Intel MCS-196/296 во строенных системах управления. - М.: Изд-во «ЭКОМ», 1997. $-688 \mathrm{c}$

26. Котельников В.А. О пропускной способности эфира и проволоки в электросвязи - Всесоюзный энергетический комитет // Материалы к I Всесоюзному съезду по вопросам технической реконструкции дела связи и развития слаботочной промышленности, 1933. Репринт статьи в журнале УФН. 2006. - T. 176. - № 7. - C. 762-770.

27. Kotelnikov V.A. The Theory of Optimum Noise Immunity. - New York: McGraw-Hill Book Co., 1959. - 140 p.

Поступила 30.08.2019 г.

\section{Информация об авторах}

Глазырин А.C., доктор технических наук, доцент отделения электроэнергетики и электротехники Инженерной школы энергетики Национального исследовательского Томского политехнического университета; профессор Института нефти и газа Югорского государственного университета.

Аникин В.B., соискатель кафедры электрической техники Омского государственного технического университета.

Буньков Д.С., аспирант отделения электроэнергетики и электротехники Инженерной школы энергетики Национального исследовательского Томского политехнического университета.

Антяскин Д.И., аспирант отделения электроэнергетики и электротехники Инженерной школы энергетики Национального исследовательского Томского политехнического университета.

Старцева Ю.Н., аспирант отделения электроэнергетики и электротехники Инженерной школы энергетики Национального исследовательского Томского политехнического университета.

Ковалев В.3., доктор технических наук, профессор Института нефти и газа Югорского государственного университета.

Хамитов P.Н., доктор технических наук, доцент, профессор кафедры электрической техники Омского государственного технического университета; профессор кафедры электроэнергетики Тюменского индустриального университета.

Кладиев $\boldsymbol{C . H . , ~ к а н д и д а т ~ т е х н и ч е с к и х ~ н а у к , ~ д о ц е н т ~ о т д е л е н и я ~ э л е к т р о э н е р г е т и к и ~ и ~ э л е к т р о т е х н и к и ~ И н ж е н е р - ~}$ ной школы энергетики Национального исследовательского Томского политехнического университета.

Филипас $\boldsymbol{A}$.A., кандидат технических наук, доцент отделения автоматизации и робототехники Инженерной школы информационных технологий и робототехники Национального исследовательского Томского политехнического университета. 
UDC 621.313.282.2: 681.5.015.7

\title{
NONLINEAR ALGEBRAIC ESTIMATION OF A VIBRATION ELECTROMAGNETIC ACTIVATOR INDUCTIVITY BY A FAILING CURRENT CURVE
}

\author{
Alexander S. Glazyrin 1,2 , \\ asglazyrin@tpu.ru \\ Vasily V. Anikin ${ }^{3}$, \\ v-anikin2012@mail.ru \\ Dmitriy S. Bunkov 1 , \\ bunkovds@tpu.ru
}

Dmitriy I. Antyaskin 1 , antyaskin.dmitriy@gmail.com

Yulia N. Startseva ${ }^{1}$, joulypaxomenko@mail.ru

Vladimir Z. Kovalev², vz_kovalev@mail.ru

Rustam N. Khamitov3,4, apple_27@mail.ru

Sergey N. Kladiev', kladiev@tpu.ru

\section{Alexander A. Filipas ${ }^{1}$,} filipas@tpu.ru

1 National Research Tomsk Polytechnic University, 30, Lenin avenue, Tomsk, 634050, Russia.

2 Yugra State University, 16, Chekhov street, Khanty-Mansiysk, 628012, Russia.

3 Omsk State Technical University, 11, Mira avenue, Omsk, 634050, Russia.

4 Tyumen Industrial University, 38, Volodarsky street, Tyumen, 625000 , Russia.

The relevance. In technologies related to preparation of drilling fluids and thinning down of highly viscous oil products, it is promisingly to use the vibration electromagnetic activators. Vibration electromagnetic activators, operating at near-resonant frequencies in limit shockfree modes with the maximum energy efficiency, requires the determination of the coil inductance at fixed values of the magnetic gap. One of the most promising methods for determining inductance is the preliminary identification of the parameters of adaptive model by falling current curve.

The main aim of the research is to develop a method of identification the inductance of a vibration electromagnetic activator coil with a fixed value of the magnetic gap based on regression analysis of the free component of the current.

Methods: ordinary differential equations, Laplace transform with zero initial conditions, transfer function impulse response, regression analysis, the methods of differential equations solving, Newton method, theory of optimization, minimization based on sum squares of residuals objective function.

Results. The paper introduces the method for preliminary identification of the inductance of vibration electromagnetic activator coils based on a regression analysis of falling current curve. The authors have concocted out the formula for objective function and composed a nonlinear algebraic equation relative to its derivative with respect to the estimated argument. The paper demonstrates the operational integrity, speed of convergence and dynamics of changing the error in the developed method with a significant deviation of the a priori values of the estimated value from the true one, both up and down. When using a 10-bit analog-to-digital converter, the algorithm for preliminary identification of inductance will require no more than 11 calculation cycles to enter the dead zone, and when using a 12-bit analog-to-digital converter, no more than 13 calculation cycles.

\section{Key words:}

Vibration electromagnetic activator, drilling fluid, highly viscous oil products, complex magnetic circuit, falling current curve, inductance estimation, regression analysis, nonlinear algebraic equation, Newton's method for solving a nonlinear equation. 


\section{REFERENCES}

1. Pevchev V.P. Principal dimensions of the short-stroke electromagnetic motor for a seismic wave generator. Journal of Mining Science, 2009, vol. 45, no. 4, pp. 372-381.

2. Chuhan Liu, Yin Zhang, Shanshan Sun, Lixin Huang, Li Yu, Xiaonan Liu, Ruiqiu Lai, Yijing Luo, Zhiyong Zhang, Zhongzhi Zhang. Oil recovery from tank bottom sludge using rhamnolipids. Journal of Petroleum Science and Engineering, 2018, vol. 170, pp. 14-20.

3. Yuhua Wang, Xiaomin Zhang, Yuying Pan, Ying Chen. Analysis of oil content in drying petroleum sludge of tank bottom. International Journal of Hydrogen Energy, 2017, vol. 42, no. 29, pp. 18681-18684.

4. Da S.L., Alves F.C., De França F.P. A review of the technological solutions for the treatment of oily sludges from petroleum refineries. Waste Management \& Research, 2012, vol. 30 (10), pp. 1016-1030.

5. Lushnikov S.V., Frank Y.A., Vorobyov D.S. Oil decontamination of bottom sediments experimental work result. Earth science research journal, 2006, vol. 10, no. 1, pp. 35-40.

6. Nekrasov V.O. Modeling bottom sediment erosion process by swirling the flow by tangential supply of oil in the tank. IOP Conference Series: Materials Science and Engineering: Transport and Storage of Hydrocarbons, 2016, vol. 154, 7 p. Available at: https://iopscience.iop.org/article/10.1088/1757 899X/154/1/012003 (accessed 13 June 2018).

7. Magalhnes S.C., Scheid C.M., CalHada L.A., Lutterbach L.M.M., Rezende R.S., Waldmann A.T.A. Real time prediction of suspended solids in drilling fluids. Review Article. Journal of Natural Gas Science and Engineering, March 2016, vol. 30, pp. 164-175.

8. Daneker V.A., Rikkonen S.V. Preparation and correction of drilling mud by technology and equipment of vibro-jet-magnetic activation. Bulletin of the Tomsk Polytechnic University. Geo assets engineering, 2017, vol. 328, no. 7, pp. 86-92. In Rus.

9. Glazyrin A.S. Sistemy pitaniya $i$ avtomaticheskogo upravleniya vibratsionnymi elektromagnitnymi aktivatorami. Diss. Kand. nauk [Power systems and automatic control of vibration electromagnetic activators. Cand. Diss.]. Tomsk, 2004. 193 p.

10. Tsurpal S.V., Glazyrin A.S. Ustroystvo dlya upravleniya elektromagnitnymi vibratorami [Device for controlling electromagnetic vibrators]. Patent RF, no. 2008102340/28, 2008

11. Gavrilin A.N., Bolovin E.V., Glazyrin A.S., Kladiev S.N., Polishchuk V.I. Resonant oscillations with a limiting amplitude in a vibration electromagnetic activator. Bulletin of the Tomsk Polytechnic University. Geo assets engineering, 2019, vol. 330, no. 1, pp. 201-213. In Rus.

12. Gavrilin A.N., Kladiev S.N., Glazyrin A.S., Bolovin E.V., Polishchuk V.I. Identification of parameters of vibration electromagnetic activator mechanical system using limiting nearresonance frequency. Bulletin of the Tomsk Polytechnic University. Geo assets engineering, 2019, vol. 330, no. 4, pp. 158-177. In Rus.

13. Maxwell J.C. An elementary treatise on electricity. Oxford, Clarendon Press, 1881. $251 \mathrm{p}$.

14. Isaev Yu.N., Vasileva O.V., Budko A.A., Lefebvre S. Parameters assessment of the inductively-coupled circuit for wireless power transfer. IOP Conference Series: Materials Science and Engineerin, 2017, vol. 177 (1), no. 012097, pp. 1-6.

15. Herbert C.R. Electromagnetic Devices. New Yorkб John Wiley \& Sons, Inc.; Londonб Chapman \& Hall, Limited, 1941. 568 p.

16. Bul B.K., Butkevich G.V., Goyello A.G., Kuraev V.G., Lysov N.E., Sakcharov P.V., Slivinskaya A.G., Taev I.S., Chu- nikchin A.A., Shopen L.V. Osnovy theorii electricheskikh apparatov [Fundamentals of the theory of electrical apparatus]. Moscow, Vysshaya shkola Publ., 1970. 600 p.

17. Godovnikov E.A., Andreeva E.G., Kovalev V.Z., Usmanov R.T Identification of parameters of power circuits pulse energy conversion systems of electromechanical equipment. Journal of Physics: Conference Series, 2019, vol. 1260 (5), no. 052007, pp. 1-8.

18. Kovalev V.Z., Arhipova O.V., Esin S.S., Tatevosyan A.A., Scherbakov A.G. Identification of mathematical models parameters of electromechanical consumers of regionally isolated electrotechnical complexes. Journal of Physics: Conference Series, 2019. vol. 1260 (5), no. 052014, pp. 1-7.

19. Polishchuk V.I., Sergeev V.L. Adaptive identification method of a signal from stray magnetic field sensor for turbogenerator diagnostics. Journal of Siberian Federal University - Mathematics and Physics, 2015, vol. 8 (2), pp. 201-207.

20. Kolomiyets E.A., Chernyshyov A.Y. Observer of rotor speed and flux is developed for the vector control system of induction motor. International Siberian Conference on Control and Communications, SIBCON 2015. Omsk, Russia, 2015. No. 7147102, pp. 1-4.

21. Tatevosyan A.S., Tatevosyan A.A., Pedder V.V., Zaharova N.V. Experimental method for identifying high-coercive permanent magnets. Journal of Physics: Conference Series, 2019, vol. 1260 (5), no. 052031, pp. 1-6.

22. Tatevosyan A.S., Tatevosyan A.A., Zakharova N.V., Lukacheva A.A. Determination of Equivalent Circuit Parameters of the Electric Pulse Device for Cleaning the Surface from Contamination. $12^{\text {th }}$ International Scientific and Technical Conference. Dynamics of Systems, Mechanisms and Machines. Dynamics 2018. Omsk, Russia, 2019. No. 8601435, pp. 1-4.

23. Kovalev A.Yu., Kuznetsov E.M., Anikin V.V. Skhemotekhnicheskoe issledovanie perekhodnoy kharakteristiki zatukhaniya toka statora pogruzhnogo asinkhronnogo elektrodvigatelya [Schematic study of the transient response of the stator current attenuation of a submersible induction motor]. Teoriya i praktika nauki tretego tysyacheletiya: sbornik statey. Ufa, RICBashgu Publ., 2014. pp. 149-152.

24. Bolovin E.V. Razrabotka algebraicheskih metodov identifikatsii parametrov asinkhronnykh dvigateley na osnove diskretnykh modeley. Diss. Kand. nauk [Development of algebraic methods for identifying the parameters of induction motors based on discrete models. Cand. Diss.]. Tomsk, 2018. 271 p.

25. Kozachenko V.F. Mikroprotsessory: rukovodstvo po primeneniyu 16-razryadnykh mikroprotsessorov Intel MCS-196/296 vo stroennykh sistemakh upravleniya [Microprocessors: a guide to using 16-bit Intel MCS-196/296 microprocessors in built-in control systems]. Moscow, EKOM Publ., 1997. 688 p.

26. Kotelnikov V.A. O propusknoy sposobnosti efira i provoloki v elektrosvyazi - Vsesoyuzny energeticheskiy komitet [On the bandwidth of ether and wire in telecommunications - All-Union Energy Committee]. Materialy k I Vsesoyuznomu sezdu po voprosam tekhnicheskoy rekonstruktsii dela svyazi i razvitiya slabotochnoy promyshlennosti [Materials to the I All-Union meeting on the issues of technical reconstruction for connection and development of lowcurrent industry]. UFN, 2006, vol. 176, no. 7, pp. 762-770.

27. Kotelnikov V.A. The theory of optimum noise immunity. New York, McGraw-Hill Book Co., 1959. 140 p.

Received: 30 August 2019.

Information about the authors

Alexander S. Glazyrin, Dr. Sc., associate professor, National Research Tomsk Polytechnic University; professor, Yugra State University.

Vasily V. Anikin, postgraduate student, Omsk state technical University.

Dmitriy S. Bunkov, postgraduate student, National Research Tomsk Polytechnic University.

Dmitriy I. Antyaskin, postgraduate student, National Research Tomsk Polytechnic University.

Yulia N. Startseva, postgraduate student, National Research Tomsk Polytechnic University.

Vladimir Z. Kovalev, Dr. Sc., professor, Yugra State University.

Rustam N. Khamitov, Dr. Sc., professor, Omsk State Technical University; professor, Tyumen Industrial University.

Sergey N. Kladiev, Cand. Sc., associate professor, National Research Tomsk Polytechnic University.

Alexander A. Filipas, Cand. Sc., associate professor, National Research Tomsk Polytechnic University. 